\title{
FCGR2A Could Function as a Prognostic Marker and Correlate with Immune Infiltration in Head and Neck Squamous Cell Carcinoma
}

\author{
Yongmei Dai $\mathbb{D}^{1},{ }^{1}$ Wenhan Chen $\mathbb{D}^{2},{ }^{2}$ Junpeng Huang $\mathbb{D}^{1},{ }^{1}$ and Tongjian Cui $\mathbb{D}^{1}$ \\ ${ }^{1}$ Department of Oncology, Shengli Clinical Medical College of Fujian Medical University \& Fujian Provincial Hospital, \\ Fujian 350001, China \\ ${ }^{2}$ The Second Clinical Medical College of Fujian Medical University, Fujian 362000, China \\ Correspondence should be addressed to Tongjian Cui; fjslctj@163.com
}

Received 27 September 2020; Accepted 17 June 2021; Published 5 July 2021

Academic Editor: Michele Rosario Colonna

Copyright (C) 2021 Yongmei Dai et al. This is an open access article distributed under the Creative Commons Attribution License, which permits unrestricted use, distribution, and reproduction in any medium, provided the original work is properly cited.

Objective. We aim to investigate the correlation between FCGR2A mRNA level and prognosis of head and neck squamous cancer (HNSC) in public databases. In addition, we investigated the correlation between FCGR2A expression and clinicopathological characteristics and tumor-infiltrating immune cells in HNSC patients. Methods. FCGR2A mRNA expression in multiple cancers was analyzed based on Gene Expression Profiling Interactive Analysis. A protein-protein interaction network was obtained based on the STRING database. The 10 proteins most closely related to FCGR2A (i.e., CD3G, PLCG2, LAT, LYN, SYK, FCGR3A, PIK3R1, HCK, ITGAM, and ITGB2) were screened, followed by establishing the protein-protein interaction network. The correlation between FCGR2A expression and immunocytes was investigated, together with the effects of FCGR2A on the metastasis, recurrence, and survival of HNSC. Results. FCGR2A expression in several carcinoma tissues was significantly higher than that of adjacent tissues. Significant differences were noticed in the HNSC samples and the adjacent tissue samples except the seven samples of grade 4 . There were statistical differences between the FCGR2A expression in tissues of grade 1, grade 2, and grade $3(P<0.05)$. In the tissues of grade 4 , the expression of FCGR2A was the lowest. The FCGR2A protein was a type of II-a receptor in $\gamma \mathrm{Fc}$ of the low-affinity immunoglobulin, which could bind with the Fc region of the immunoglobulin $\gamma$. There was a correlation between the FCGR2A gene and the distal HNSC metastasis. FCGR2A gene expression was correlated with the survival and prognosis. The GSE65858 dataset was selected for the validation. The FCGR2A expression was significantly correlated with total survival $(P=0.0107)$ and progression-free survival $(P=0.0362)$. Conclusions. Our findings shed light on the importance of FCGR2A in HNSC and illustrated a potential relationship between FCGR2A and tumor-immune interactions.

\section{Introduction}

Head and neck squamous cancer (HNSC), one of the most common types of human malignancy, shows an annual incidence of more than 780,000 cases worldwide [1-3]. To date, the treatment options of HNSC include surgery, radiation, and chemotherapy; however, it leads to a mortality rate of up to $50 \%$ [4]. Currently, most of the studies on HNSC are mainly focused on disease prevention, early screening, identification of target molecules, and the establishment of new therapies. To our best knowledge, there are still some disadvantages in the prediction of prognosis among HNSC patients. Therefore, it is urgent to screen specific and sensitive biomarkers for the evaluation of an individualized treatment regimen.

The FCGR2A gene, responsible for encoding the Fc $\gamma$ RIIa protein, is localized at 1q23 [5] consisting of 10 exons and 9 introns. It is a member of the immunoglobulin Fc receptor (FCGR) gene on the surface of cells involved in immune responses. Based on its function, Fc $\gamma$ RIIa is divided into two subtypes including Fc $\gamma$ RIIa and Fc $\gamma$ RIIb, which are classified as IgG receptors with low to moderate affinity. It is mainly involved in binding with IgG, and is expressed in the neutrophils, monocytes, macrophages, dendritic cells 


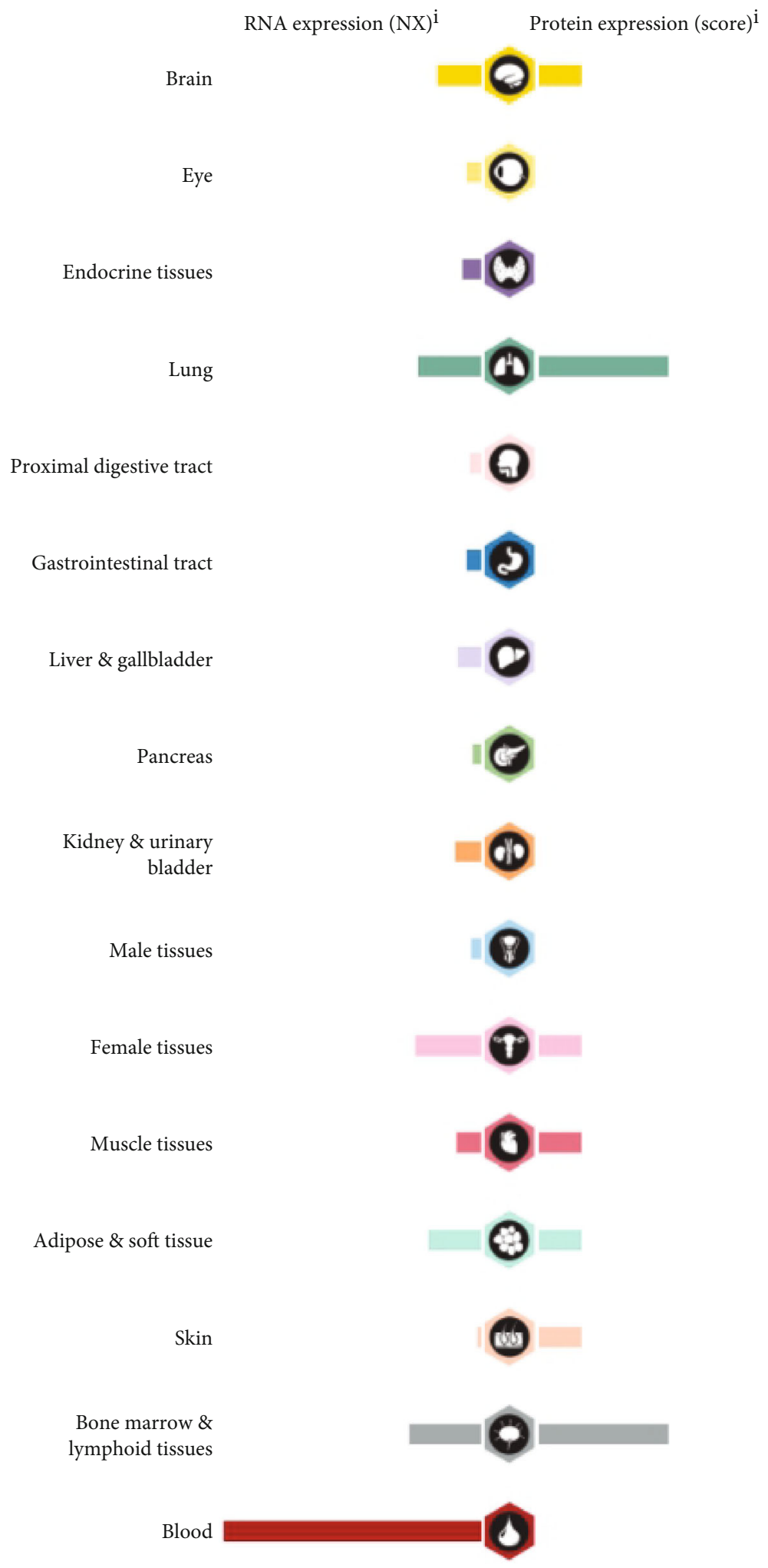

FIGURE 1: Expression of FCGR2A mRNA and protein expression in normal tissues.

(DCs), B lymphocytes, and platelets. In a previous study, FCGRs generated by the natural killer (NK) cells, macrophages, and DCs could modulate the antibody-dependent cytotoxicities, which were crucial for the elimination of can- cer cells. FCGR2A gene polymorphism was reportedly associated with the susceptibility of inflammation-related diseases, such as atherosclerosis [6], Takayasu arteritis [7], systemic lupus erythematosus (SLE) [8], and ulcerative colitis [9]. To 


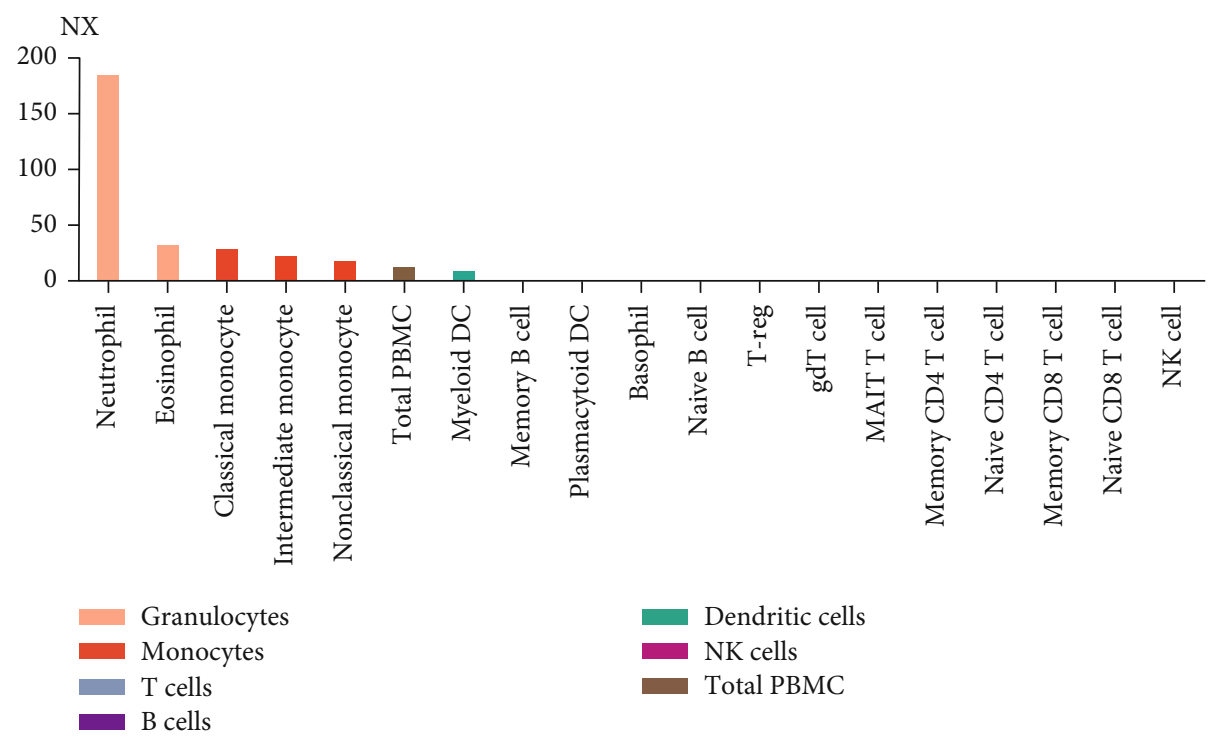

FIGURE 2: FCGR2A mRNA-enriched cell types in blood samples.

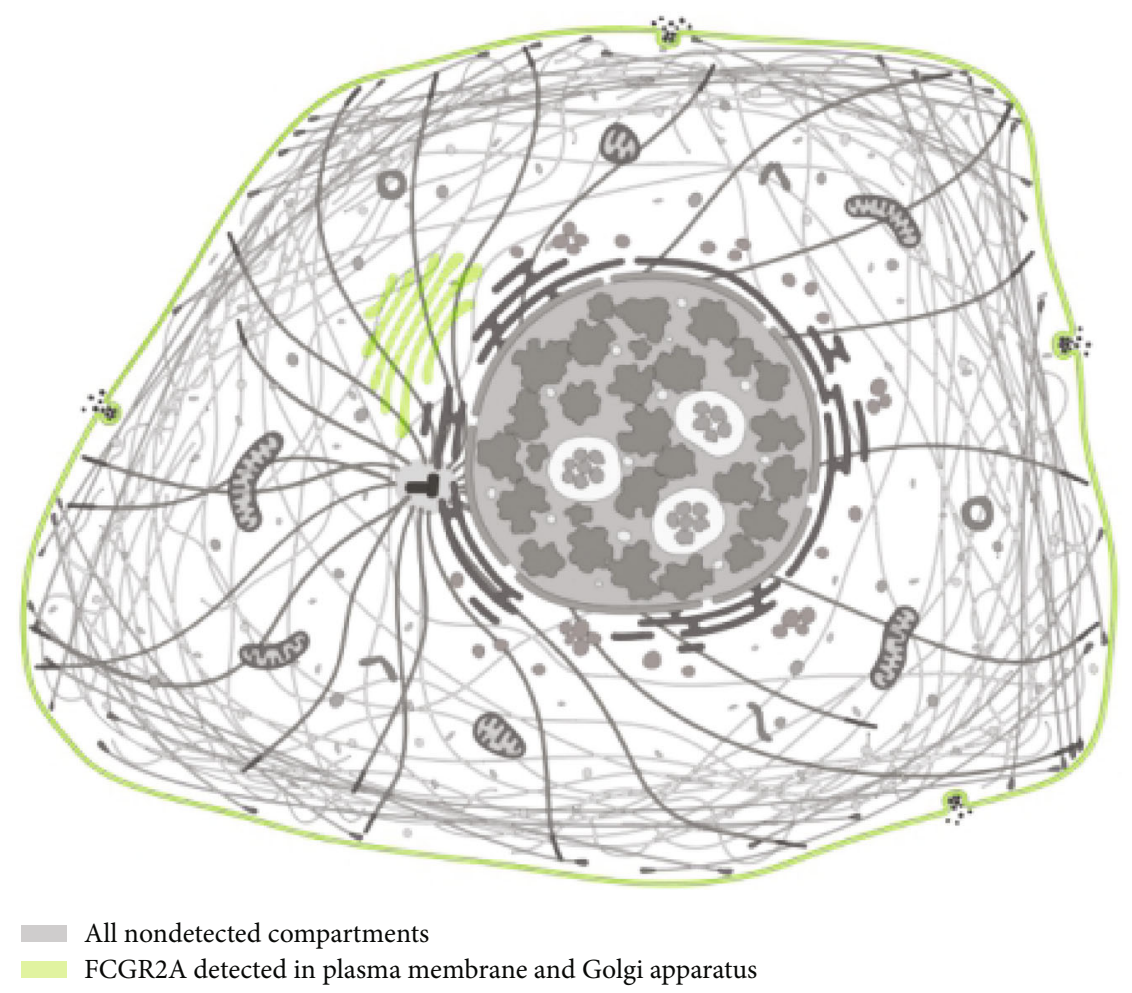

FIGURE 3: Expression of FCGR2A in Golgi body and plasma membrane.

date, some studies have been conducted to investigate the roles of FCGR2A gene polymorphism in malignancies. In a previous study [10], the FCGR2A genotype was associated with the treatment efficiency of colorectal cancer patients who received cetuximab administration, which triggered no additional toxicities. To our best knowledge, few studies focused on the investigation on the oncogenic roles of the FCGR2A gene in HNSC in a systematic manner. In this study, we evaluated the association between FCGR2A
mRNA level and HNSC prognosis in public databases such as TCGA, TISCH, Exocarta, and CPTAC. Moreover, we investigated the correlation between FCGR2A expression and clinicopathological characteristics or the tumorinfiltration in HNSC patients. Our findings shed light on the importance of FCGR2A in HNSC and contributed to the investigation on the potential relationship between FCGR2A and tumor-immune interaction and its underlying mechanism. 


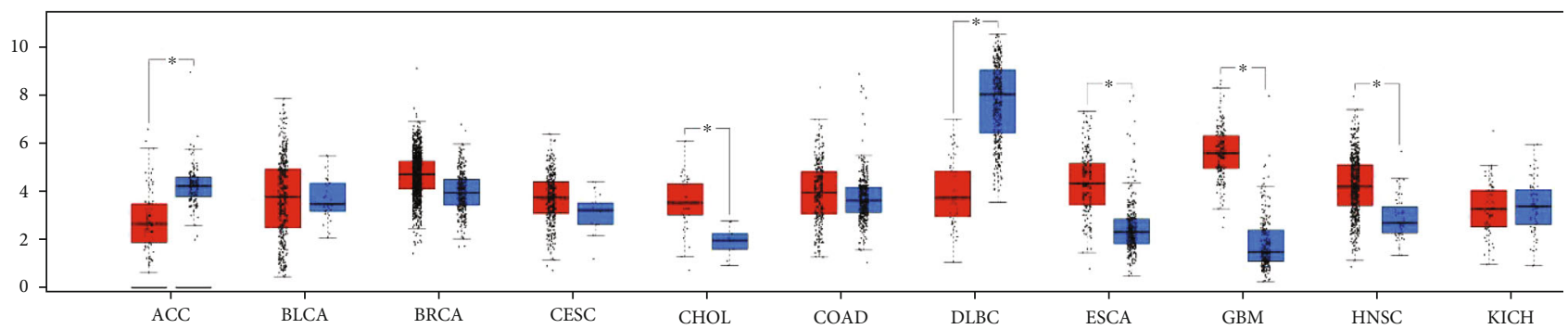

(a)

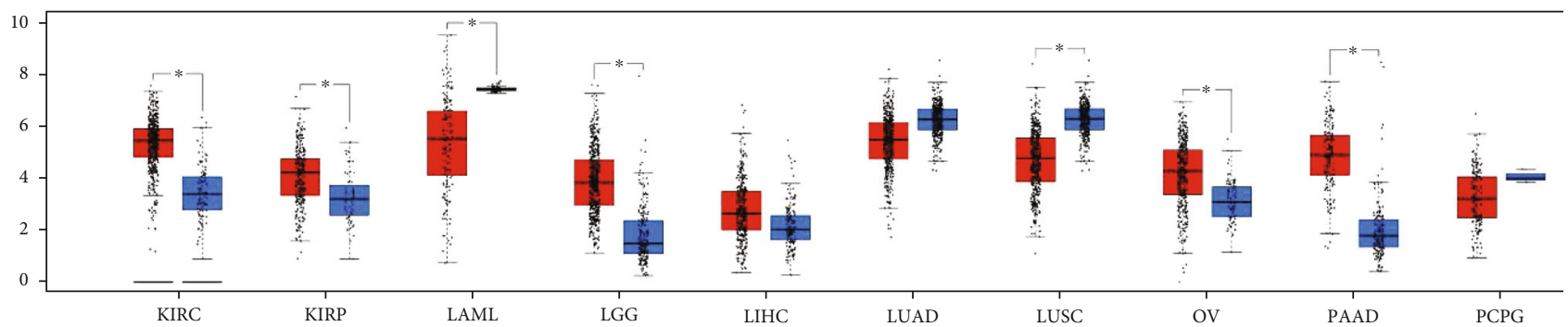

(b)

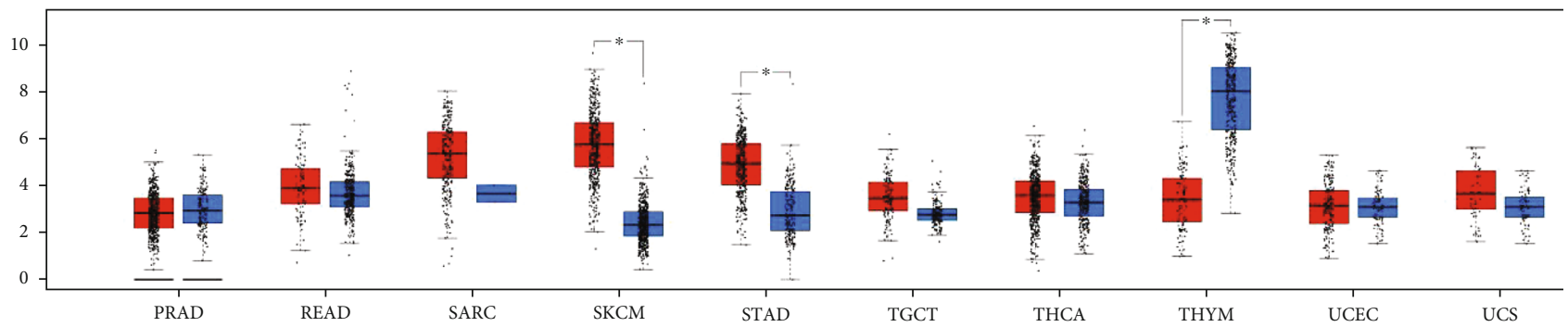

(c)

FIGURE 4: Expression of FCGR2A in tumor (red column) and adjacent tissues (blue column). ${ }^{*} P<0.05$. ACC: adrenocortical carcinoma; BLCA: bladder urothelial carcinoma; BRCA: breast invasive carcinoma; CESC: cervical squamous cell carcinoma and endocervical adenocarcinoma; CHOL: cholangiocarcinoma; COAD: colon adenocarcinoma; DLBC: lymphoid neoplasm diffuse large B cell lymphoma; ESCA: esophageal carcinoma; GBM: glioblastoma multiforme; HNSC: head and neck squamous carcinoma; KICH: kidney chromophobe; KIRC: kidney renal clear cell carcinoma; KIRP: kidney renal papillary cell carcinoma; AML: acute myeloid leukemia; LGG: brain lowergrade glioma; LIHC: liver hepatocellular carcinoma; LUAD: lung adenocarcinoma; LUSC: lung squamous cell carcinoma; OV: ovarian serous cystadenocarcinoma; PAAD: pancreatic adenocarcinoma; PCPG: pheochromocytoma and paraganglioma; PRAD: prostate adenocarcinoma; READ: rectum adenocarcinoma; SARC: sarcoma; SKCM: skin cutaneous melanoma; STAD: stomach adenocarcinoma; TGCT: testicular germ cell tumors; THCA: thyroid carcinoma; THYM: thymoma; UCEC: uterine corpus endometrial carcinoma; UCS: uterine carcinosarcomas.

\section{Materials and Methods}

2.1. Expression of FCGR2A $m R N A$. The Human Protein Atlas database (http://www.proteinatlas.org/) was utilized to analyze the expression of FCGR2A in multiple cancers. In addition, the expression profile of the mRNA and protein in the normal tissues and cells was analyzed. Gene Expression Profiling Interactive Analysis (GEPIA, http://gepia.cancer-pku $. \mathrm{cn} /$ ) was utilized to analyze the expression differences of FCGR2A in different cancers and the adjacent cancer tissues, followed by depicting the plot. Moreover, the expression of FCGR2A in HNSC was explored using UALCAN dataset (http://ualcan.path.uab.edu) based on the gender, age, and other classification standards.

2.2. FCGR2A Protein Interaction Network and the Gene Enrichment Analysis. The protein-protein interaction net- work was obtained based on the STRING database. On this basis, the top 10 proteins related to FCGR2A (CD3G, PLCG2, LAT, LYN, SYK, FCGR3A, PIK3R1, HCK, ITGAM, and ITGB2) were screened, followed by establishing the protein-protein interaction network. In the homepage of Metascape (http://metascape.org), enrichment analysis was performed after entering the 11 genes including FCGR2A, CD3G, PLCG2, LAT, LYN, SYK, FCGR3A, PIK3R1, HCK, ITGAM, and ITGB2. Then, KEGG pathway analysis, GO analysis, and gene sets were integrated and visualized. Finally, the 11 genes encoding these proteins including FCGR2A were entered into Metascape.

2.3. Correlation between FCGR2A Expression and Immunocytes. The TIMER database [11] (http://cistrome .dfci.harvard.edu/TIMER/) was utilized to determine the association between FCGR2A expression and the immune 


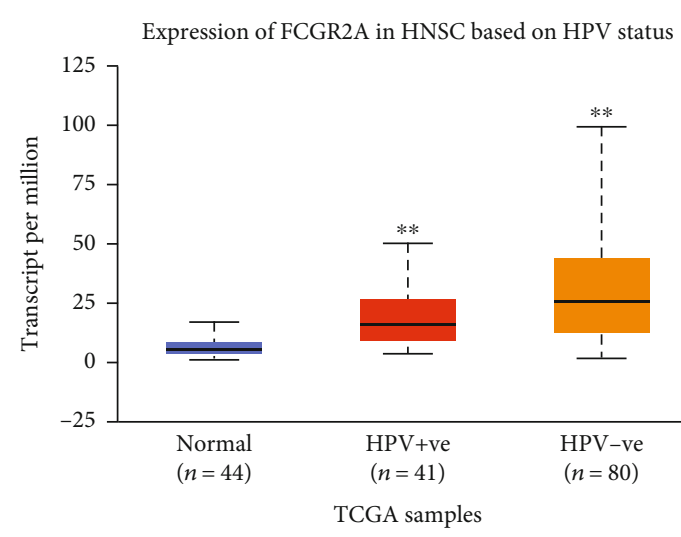

(a)

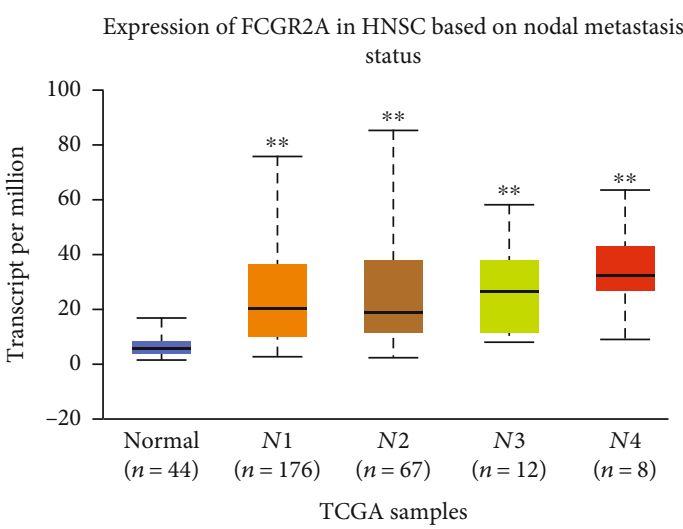

(c)

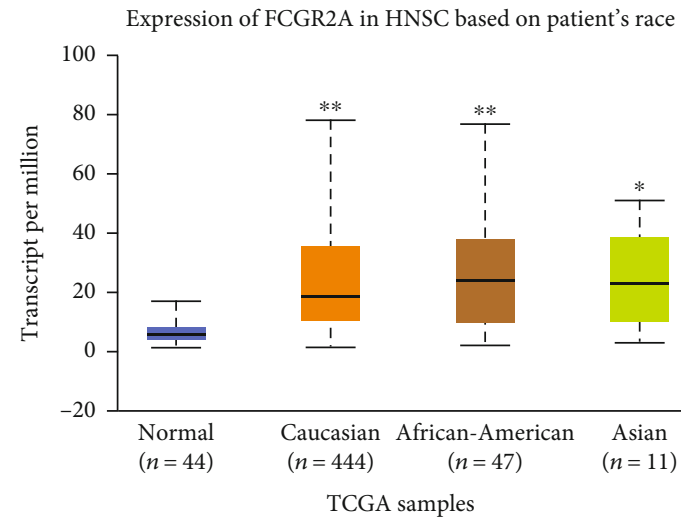

(e)

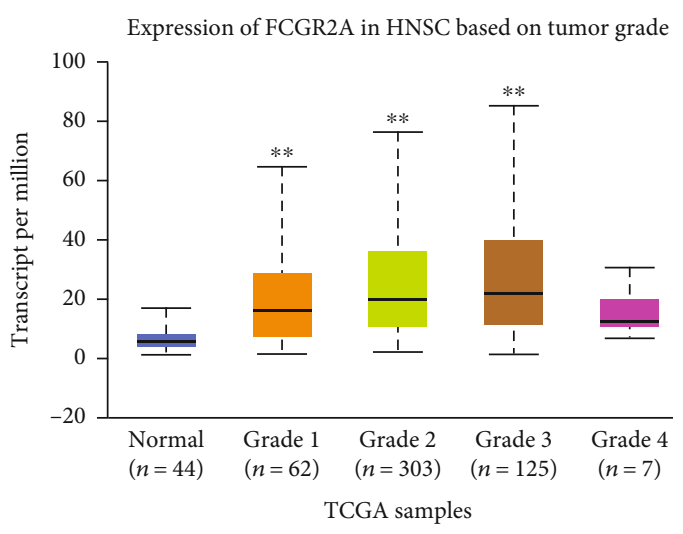

(b)

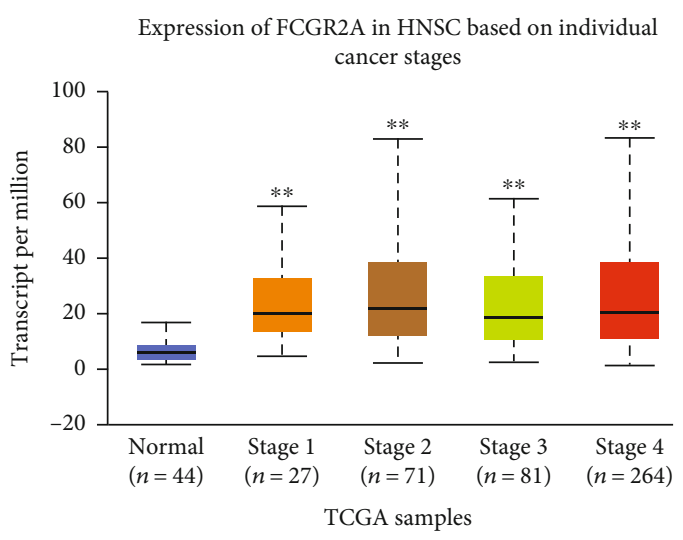

(d)

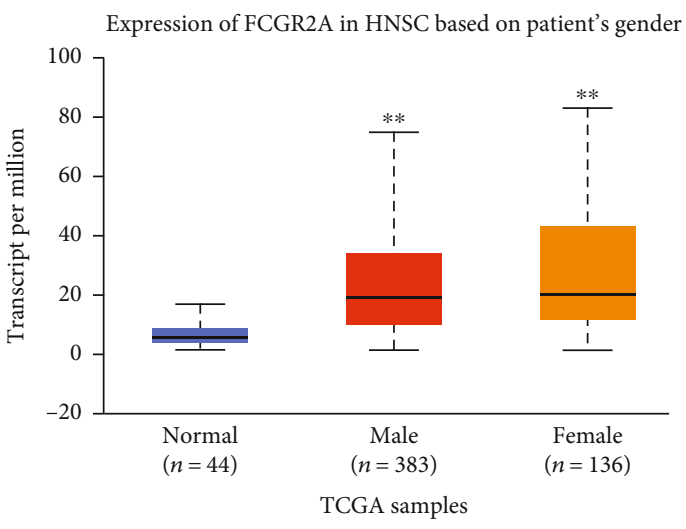

(f)

FIGURE 5: FCGR2A transcription in subgroups of patients with HNSC, stratified based on gender, race, and other criteria (UALCAN). (a) Relative expression of FCGR2A in normal and HPV-positive and HPV-negative HNSC samples. (b) Relative expression of FCGR2A in normal and HNSC patients with grade 1,2,3, and 4 tumors. (c) Relative expression of FCGR2A in normal or HNSC patients with stage N1, N2, N3, and N4. (d) Relative expression of FCGR2A in normal patients or in HNSC patients at stages 1, 2, 3, and 4. (e) Relative expression of FCGR2A in normal, Caucasian, African-American, and Asian HNSC patients. (f) Relative expression of FCGR2A in normal, male, and female HNSC patients. The central marker was the median; the edges of the box were the 25th and 75th percentiles. The $t$-test was used to estimate the significance of difference in gene expression levels between groups. ${ }^{*} P<0.05 ;{ }^{* *} P<0.01 ;{ }^{* * *} P<0.001$.

infiltration. To begin with, the purity estimation was acquired using the tool CHAT [12] and validated by a diluted series with known tumor/normal mixture ratios. On this basis, we established the correlation between FCGR2A expression and tumor purity. Pearson's correlation was utilized for the analysis. Next, FCGR2A-expressing tumors in HNSC showed various immunocyte infiltration levels, including B lymphocytes, CD4+ T lymphocytes, CD8+ T lymphocytes, neutrophils, macrophages, and DCs.

2.4. Effects of FCGR2A on the Metastasis, Recurrence, and Survival of HNSC. To investigate the prognostic role of 

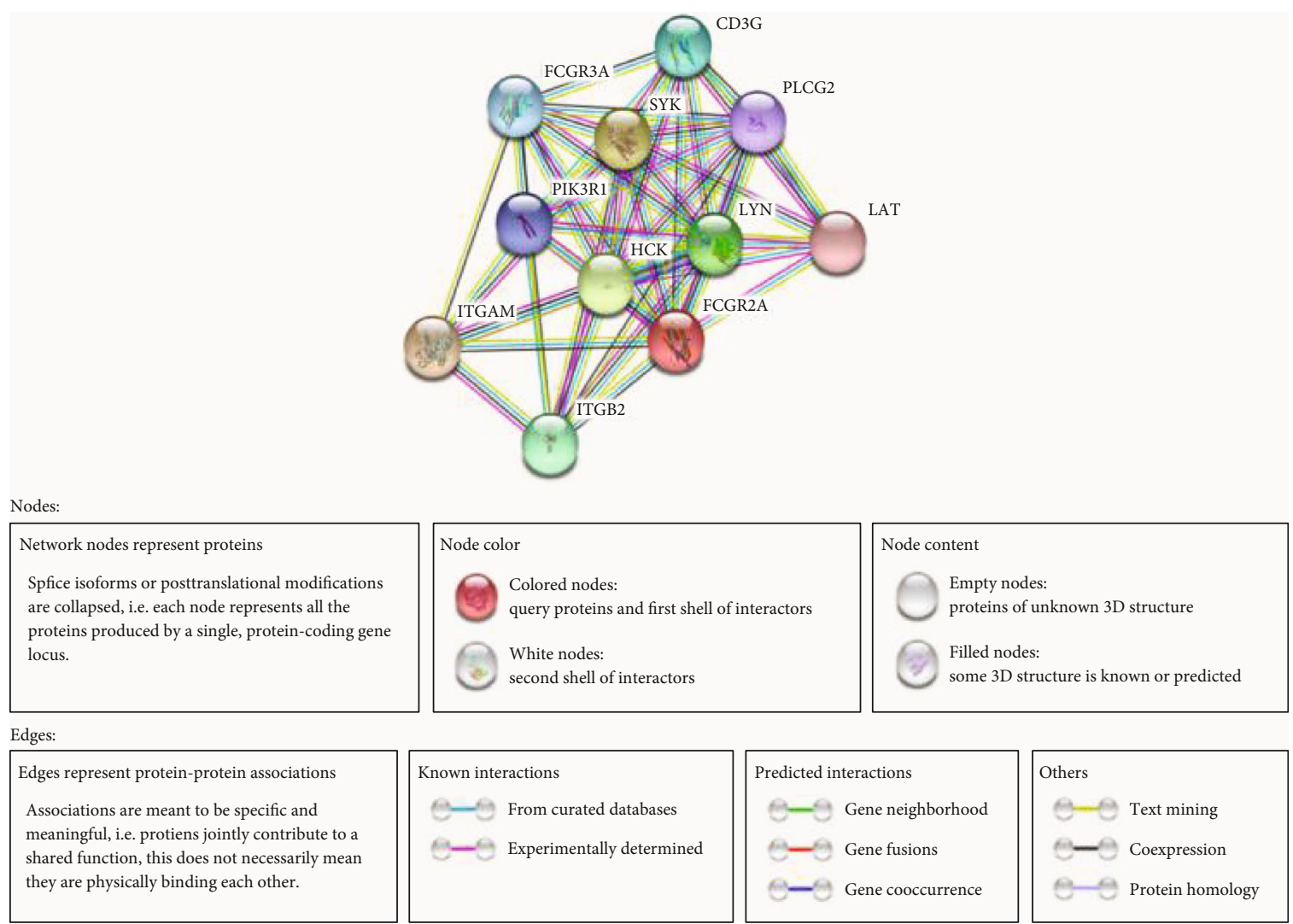

FIGURE 6: FCGR2A-related protein interaction network.

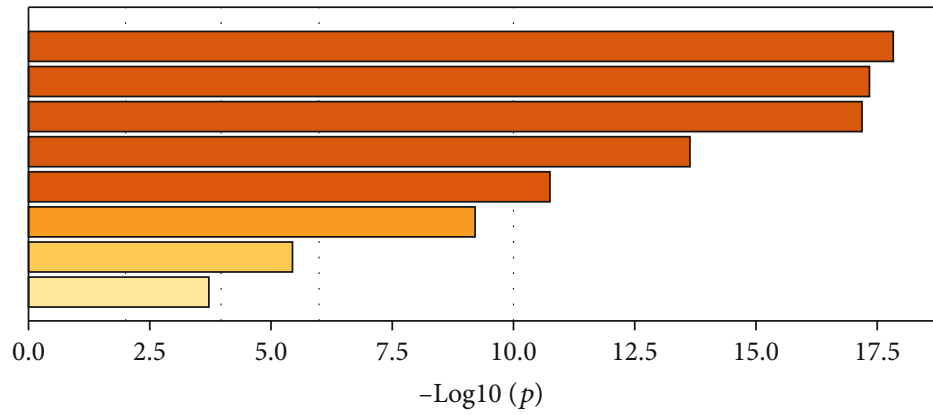

R-HSA-2029481: FCGR activation

GO:0002757: immune response-activating signal transduction GO:0006909: phagocytosis M141: PID PI3KCl pathway M50: PID PTP1B pathway GO:0007229: integrin-mediated signaling pathway R-HSA-388841: costimulation by the CD28 family GO:0009617: response to bacterium

FIgURE 7: Heat map of enrichment of FCGR2A-related protein genes. The shade of the color depended on the $P$ value.

FCGR2A mRNA in HNSC, the Kaplan-Meier plotter [13] (http://www.kmplot.com) was used to determine the prognostic significance. Based on the GEOquery package, the HNSC GSE65858 was downloaded from the GEO database [14] for the prognosis analysis. There were 253 tumor samples in the GSE65858 database, among which 179 were human papilloma virus- (HPV-) negative samples. The sequencing platform was Illumina HumanHT-12 v4.0. Downloaded from The Cancer Genome Atlas (TCGA) database, the Long-term Outcome and Gene Expression Profiling Database of Pancancer (LOGpc) platform was used for analyzing the overall survival (OS) and disease-free survival (DSS) of HNSC patients.
2.5. Statistical Analysis. Kaplan-Meier method was used for estimating the survival curve. To compare the survival curve, the log-rank test was utilized for the hazard ratio (HR) and the $P$ value of the Kaplan-Meier plotter and GEPIA. Spearman's analysis was utilized for the analysis of the correlation of genes.

\section{Results}

3.1. Expression of FCGR2A $m R N A$. According to the Human Protein Atlas website, the FCGR2A gene was expressed in various tissues. Serum FCGR2A mRNA was the highest among these tissues (Figure 1). The enriched cell types of 

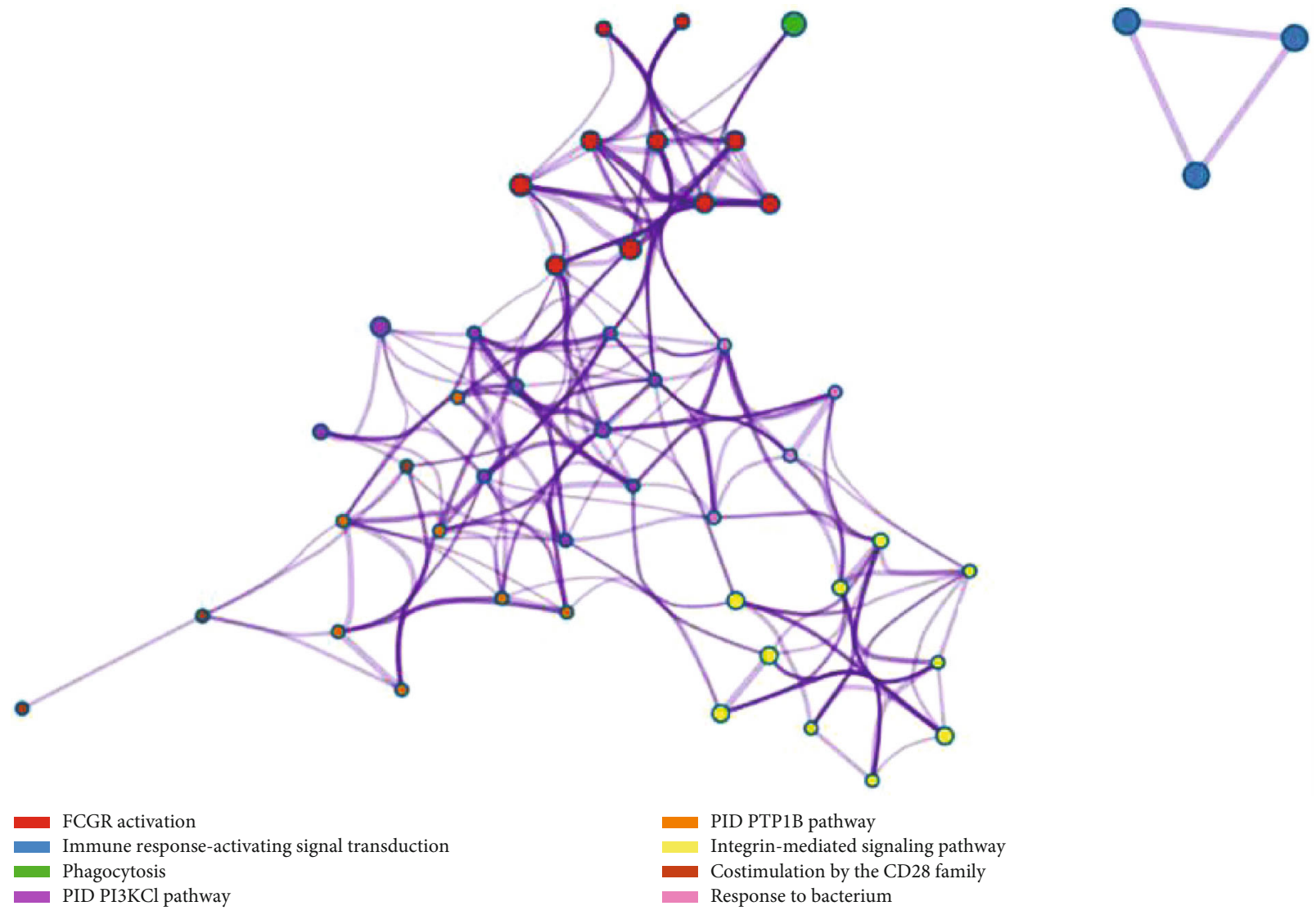

(a)
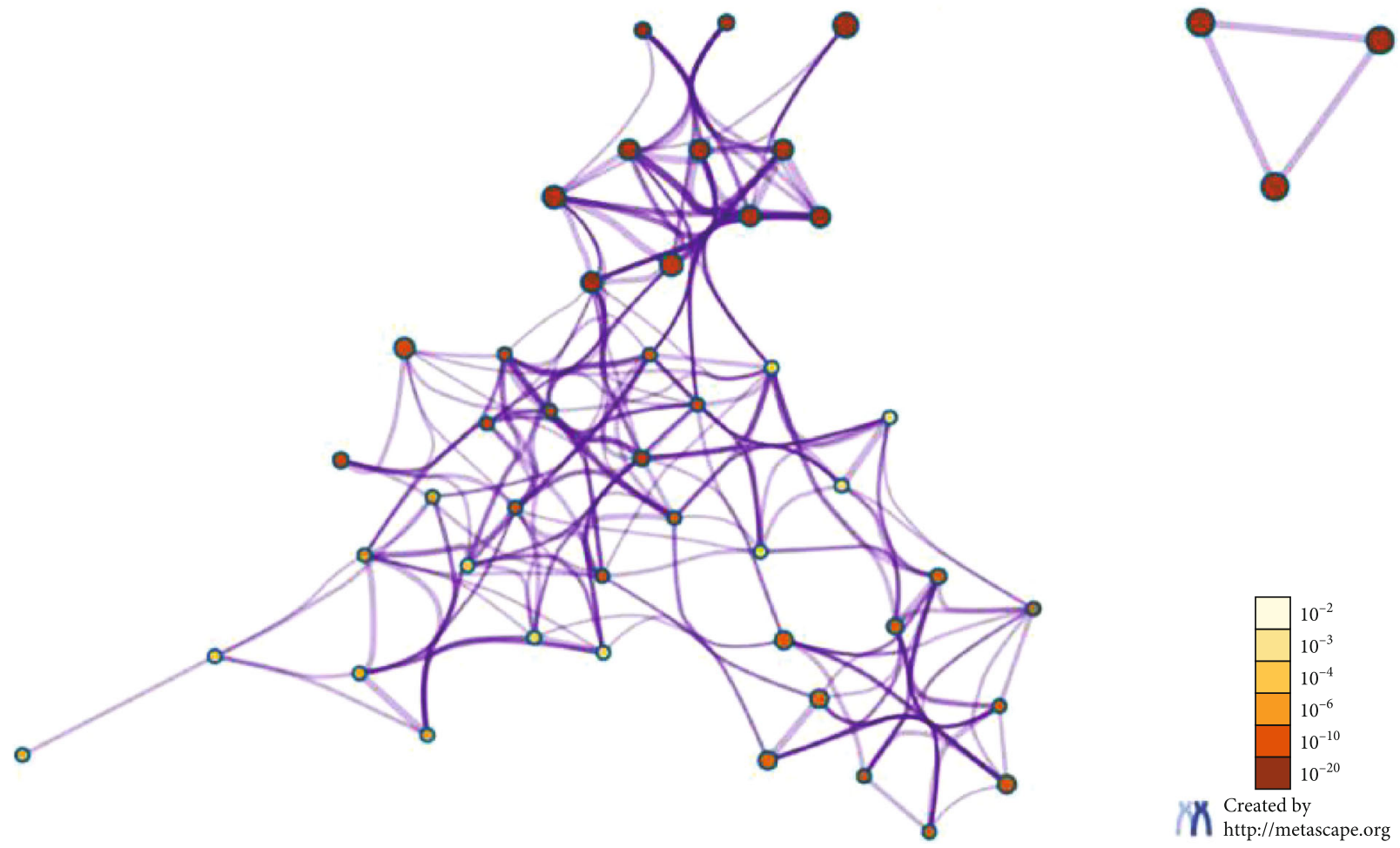

(b)

FIGURE 8: Network diagram of enrichment of gene cluster. (a) Different colors according to enrichment item markers of different functions. (b) Different color shades according to $P$ values. The size of each node was proportional to the number of input genes. 
TABLE 1: Correlation between FCGR2A copy number variation and immune infiltration abundance in HNSCC.

\begin{tabular}{|c|c|c|c|c|}
\hline Variable & CNA level & $P$ value, HNSCC & $P$ value, HNSCC-HPVneg & $P$ value, HNSCC-HPVpos \\
\hline \multirow{4}{*}{ B cell } & Arm-level deletion & $6.52 E-08$ & $2.26 E-04$ & 1 \\
\hline & Diploid/normal & 1 & 1 & 1 \\
\hline & Arm-level gain & $1.40 E-01$ & $6.70 E-01$ & $1.30 E-01$ \\
\hline & High amplication & $8.60 E-01$ & $6.32 E-01$ & \\
\hline \multirow{4}{*}{ CD8+ T cell } & Arm-level deletion & $1.33 E-06$ & $9.28 E-05$ & 1 \\
\hline & Diploid/normal & 1 & 1 & 1 \\
\hline & Arm-level gain & $4.48 E-05$ & $1.64 E-04$ & $6.00 E-02$ \\
\hline & High amplication & $3.35 E-01$ & $7.26 E-01$ & \\
\hline \multirow{4}{*}{ CD4+ T cell } & Arm-level deletion & $2.26 E-03$ & $7.29 E-02$ & 1 \\
\hline & Diploid/normal & 1 & 1 & 1 \\
\hline & Arm-level gain & $4.41 E-02$ & $8.43 E-02$ & $3.62 E-01$ \\
\hline & High amplication & $3.22 E-02$ & $1.57 E-01$ & \\
\hline \multirow{4}{*}{ Macrophage } & Arm-level deletion & $7.85 E-04$ & $1.81 E-03$ & 1 \\
\hline & Diploid/normal & 1 & 1 & 1 \\
\hline & Arm-level gain & $8.84 E-02$ & $2.36 E-02$ & $6.15 E-01$ \\
\hline & High amplication & $7.60 E-01$ & $7.81 E-01$ & \\
\hline \multirow{4}{*}{ Neutrophil } & Arm-level deletion & $6.85 E-03$ & $1.20 E-02$ & 1 \\
\hline & Diploid/normal & 1 & 1 & 1 \\
\hline & Arm-level gain & $3.85 E-07$ & $1.34 E-05$ & $1.57 E-02$ \\
\hline & High amplication & $1.50 E-02$ & $1.82 E-02$ & \\
\hline \multirow{4}{*}{ Dendritic cell } & Arm-level deletion & $5.29 E-05$ & $7.38 E-04$ & 1 \\
\hline & Diploid/normal & 1 & 1 & 1 \\
\hline & Arm-level gain & $1.17 E-03$ & $1.51 E-02$ & $3.78 E-02$ \\
\hline & High amplication & $8.90 E-01$ & $9.60 E-01$ & \\
\hline
\end{tabular}

HNSCC: head and neck squamous cell carcinoma; HNSCC-HPVneg: HPV-negative, head and neck squamous cell carcinoma; HNSCC-HPVpos: HPVpositive, head and neck squamous cell carcinoma.

the blood expressing the FCGR2A gene included neutrophils, eosinophils, and monocytes (Figure 2). In the cellular views, FCGR2A was mainly expressed in the Golgi body and the cell membrane (Figure 3).

In the GEPIA website, we compared the expression of FCGR2A in the adjacent cancer tissues and the cancer tissues. Expression of FCGR2A in the carcinoma of the bile duct, carcinoma of the esophagus, the glioblastoma multiforme, HNSC, the clear cell carcinoma of the kidney, renal papillary cell carcinoma, low-grade cerebral glioma, ovary serous cystadenocarcinoma, carcinoma of the pancreas, melanoma, and gastric cancer tissues was significantly higher than that of the corresponding adjacent tissues. In contrast, the expression of FCGR2A in the adrenocortical carcinoma, diffuse large $B$ cell lymphoma, acute myeloid leukemia, pulmonary squamous carcinoma, and thymus cancer tissues was significantly lower than that of the corresponding adjacent tissues (Figure 4).

3.2. Expression of FCGR2A in HNSC. We compared the HNSC samples with the adjacent tissues based on the UALCAN website and tried to investigate the correlation between
FCGR2A expression and the clinicopathological factors. As shown in Figure 5, significant differences were noticed in the HNSC samples and the adjacent tissue samples except the seven samples of grade 4, based on various clinicopathological factors. Compared with HPV-positive samples, the FCGR2A expression in the HPV-negative HNSC patients showed a significant increase $(P<0.05)$. The expression of FCGR2A was associated with the tumor grading, in a gradedependent manner. There were statistical differences between the FCGR2A expression in tissues of grade 1, grade 2 , and grade $3(P<0.05)$. In the tissues of grade 4 , the expression of FCGR2A was the lowest. However, there were only seven samples, and we could not evaluate the reliability of the results. There were no statistical differences in the expression of FCGR2A among the samples obtained from cases of different gender, race, and tumor staging $(P>0.05)$.

3.3. FCGR2A Protein Interaction Network and Gene Enrichment Analysis. Upon entry of FCGR2A into the STRING website, the gene database of Homo sapiens was selected. The data indicated that the FCGR2A protein was a type of II-a receptor in the $\gamma \mathrm{Fc}$ of the low-affinity 

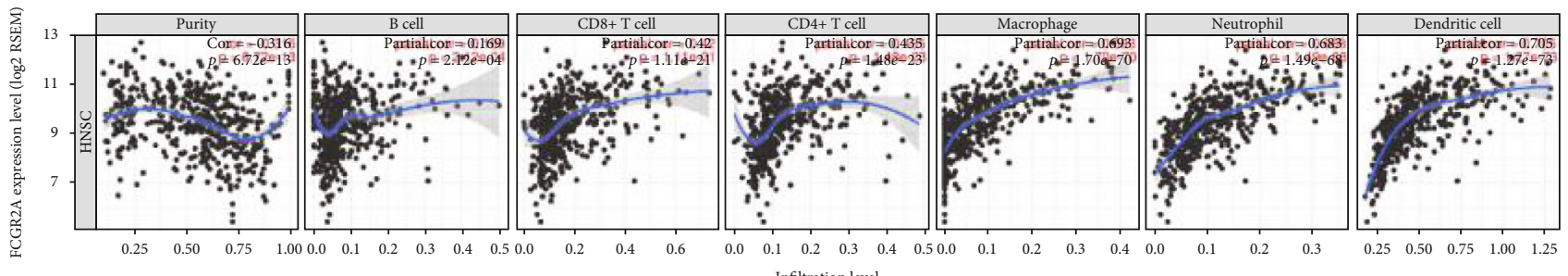

(a)
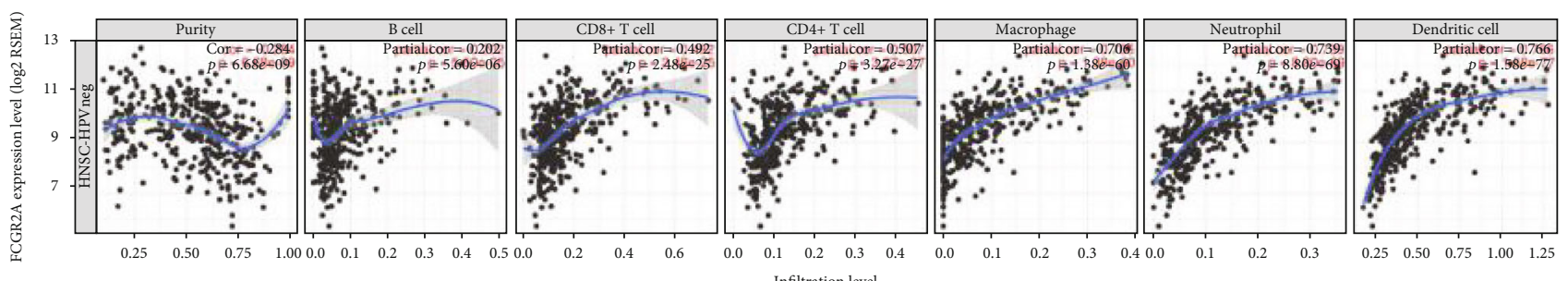

(b)
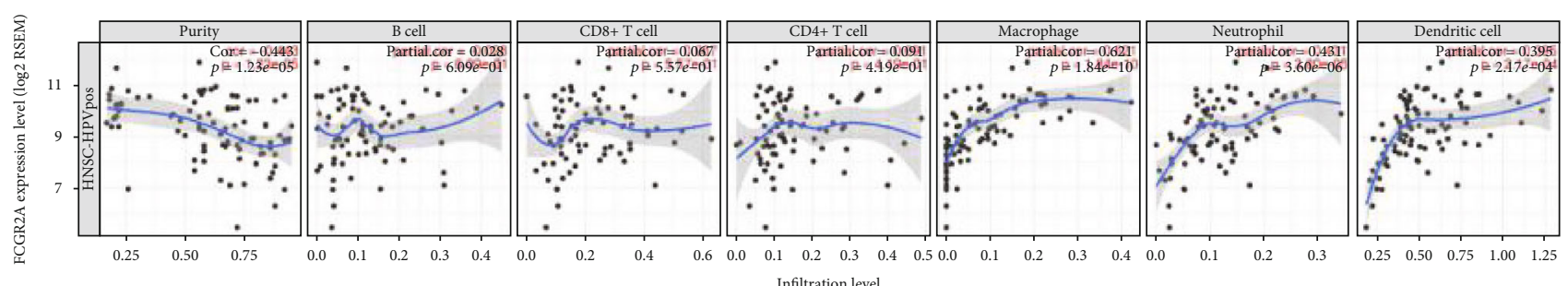

(c)

FIGURE 9: Correlation of FCGR2A expression with immune infiltration level in the TIMER database. (a) All head and neck cancer samples. (b) HPV-negative HNSC samples. (c) HPV-positive HNSC samples. The blue curve was the fitting curve of the scatter plot.

immunoglobulin, which could bind with the Fc region of the immunoglobulin $\gamma$. It could promote the phagocytosis of the antigens. Besides, it triggered the cellular responses targeting the pathogens and soluble antigens through binding with the IgG.

We then screened the top 10 proteins that were closely related to FCGR2A including CD3G, PLCG2, LAT, LYN, SYK, FCGR3A, PIK3R1, HCK, ITGAM, and ITGB2. On this basis, the protein-protein interaction network was established (Figure 6). Subsequently, the genes encoding the 11 proteins including the top 10 proteins and the FCGR2A protein were entered into the Metascape website, using $H$. sapiens as the type. Initially, we confirmed all the enriched items that were subject to statistical analysis, such as GO/KEGG, the typical signaling pathway, and hallmark gene sets. The enriched plot was obtained based on the $P$ value and enriched factors (Figure 7). Afterwards, the enrichment plot was transmitted to the network topology. Each item was represented by a circle node. Its size was positively correlated to the gene amount. The items with a similarity score of $>0.3$ were linked by a line, of which the line thickness represented the similarity score (Figure 8 ).

3.4. Correlation between Expression of FCGR2A and Immunocytes in TIMER. In this study, the TIMER database was utilized to investigate the correlation between FCGR2A expression and immune infiltration of HNSC (Table 1). The scatter plot displayed the corrected partial Spearman's correlation and the statistical significance. On this basis, there was a negative correlation between FCGR2A expression and the HNSC purity. Meanwhile, there were significant differences in the 6 types of immunocytes and FCGR2A expression $(P<0.05)$, especially the DCs, neutrophils, and macrophages. There was a correlation between FCGR2A expression and the 6 infiltrating immunocytes regardless of the HPV negativity or positivity (Figure 9). This implied that FCGR2A expression was correlated with the immune infiltration of HNSC.

The SCNA model was utilized to explore the association between the FCGR2A gene copy number variation (CNV) and the richness of the immune infiltration. Based on the CNA, we determined the infiltration of 6 types of immunocytes. GISTIC 2.0 served as the grouping standards for the CNA which were divided into 5 types including deep deletion (-2), arm-level deletion (-1), diploid/normal (0), arm-level gain (1), and high amplification (2). Except the CD4+ T lymphocytes from the HPV-negative samples, there was a significant correlation between the FCGR2A expression and arm deletion in the immunocytes in all the HNSC samples and HPV-negative HNSC samples. In contrast, there was no correlation between the FCGR2A expression and arm deletion in the immunocytes between all the HNSC samples and HPV- 


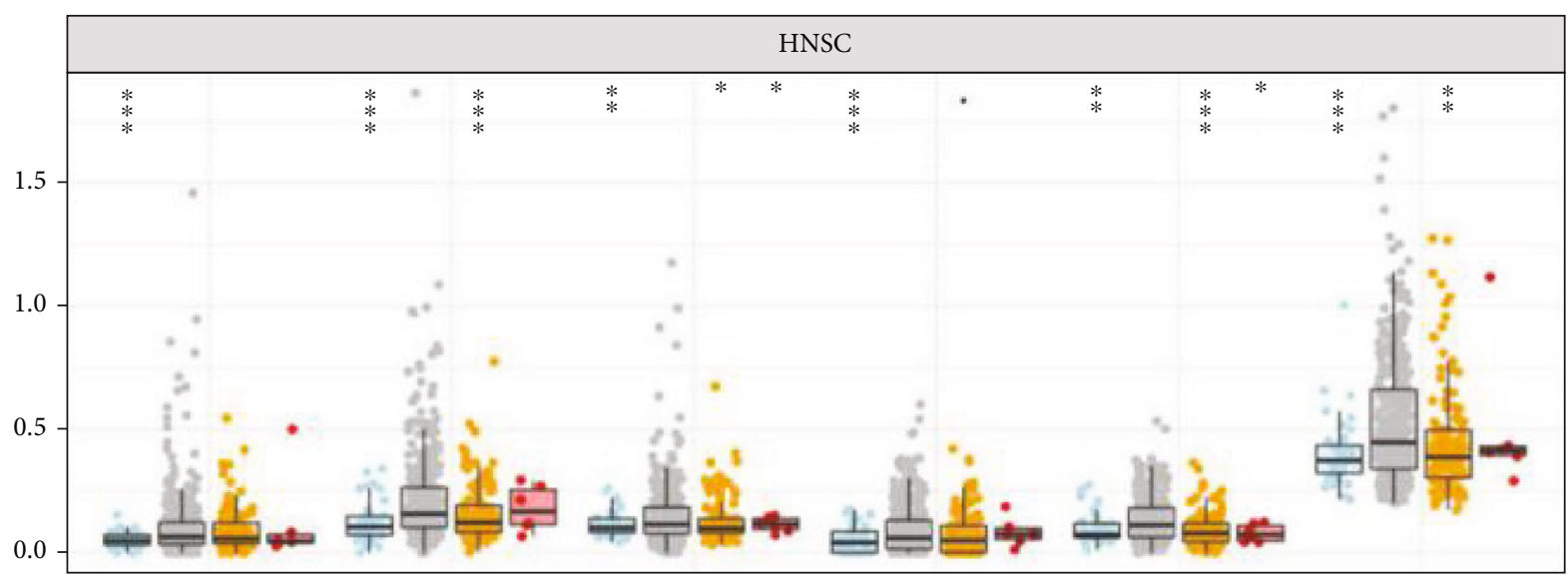

Copy number

Arm-level deletion

† Diploid/normal

Arm-level gain

High amplication

(a)

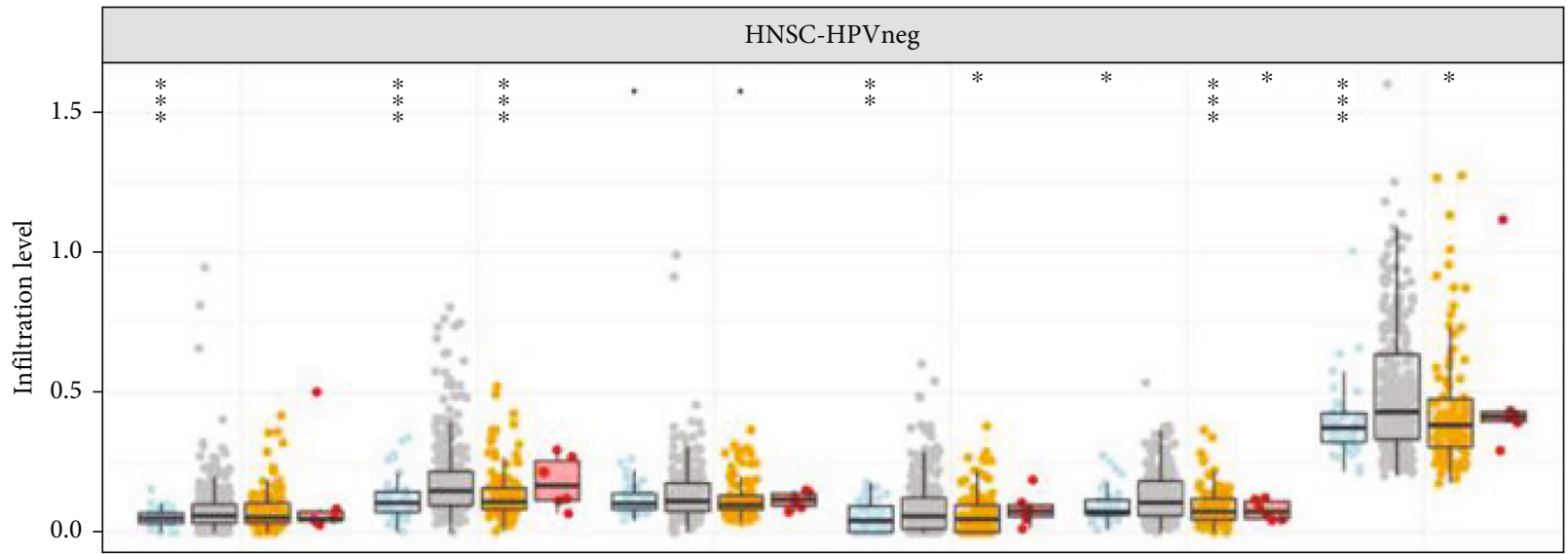

Copy number

Arm-level deletion

Diploid/normal

Arm-level gain

High amplication

(b)

Figure 10: Continued. 


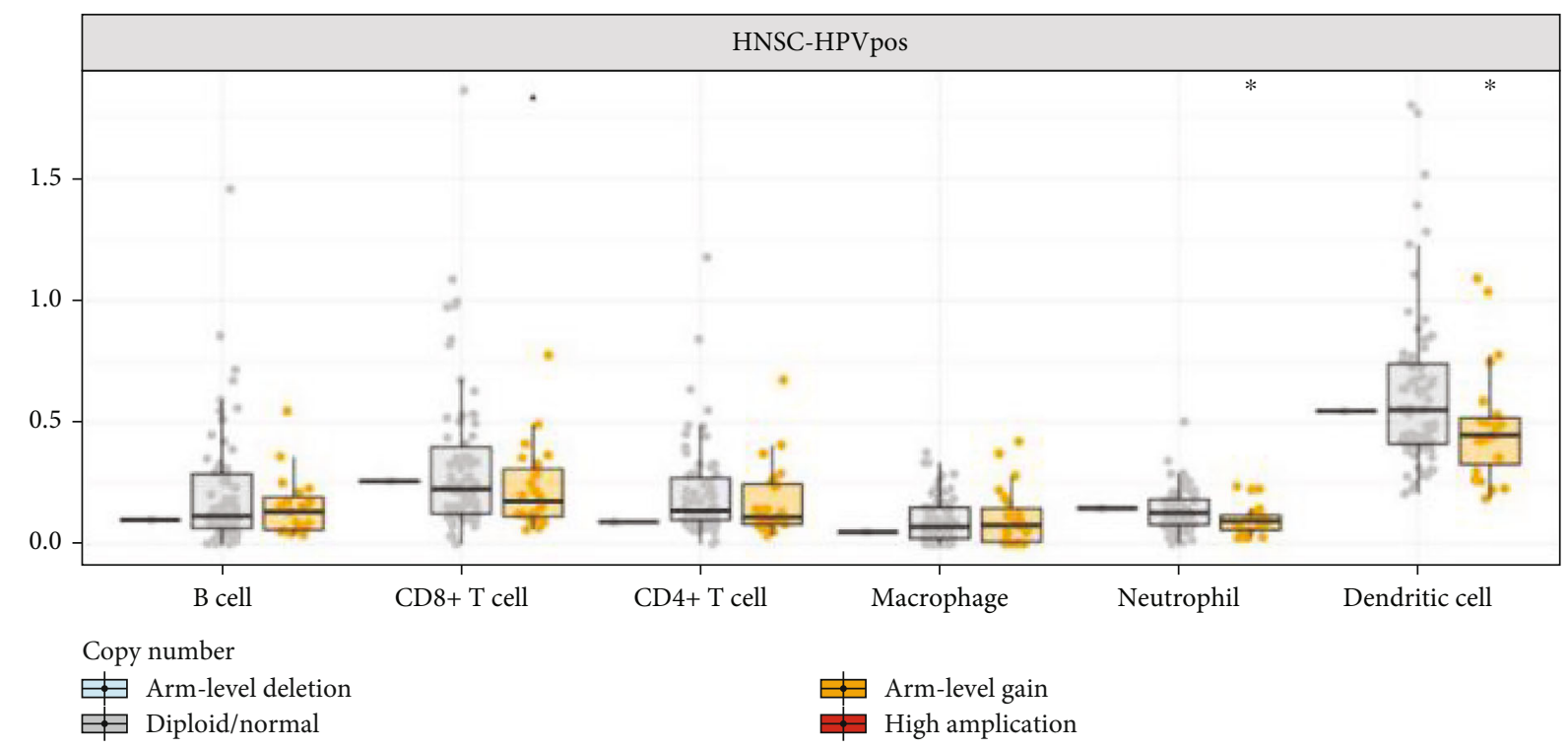

(c)

FIGURE 10: SCNA model of FCGR2A gene copy number variation and immunoinfiltration abundance. (a) All head and neck cancer samples. (b) HPV-negative HNSC samples. (c) HPV-positive HNSC samples. ${ }^{*} P<0.05$; ${ }^{* *} P<0.01$; ${ }^{* * *} P<0.001$.

positive HNSC samples. As shown in Figure 10, the infiltration of DCs was higher in the cells with no anomaly in the chromosome structure or those with enlarged arms.

To further investigate the correlation between FCGR2A and various immune infiltration cells, TIMER was used to investigate the correlation between FCGR2A expression and the immune-labeled genes (Table 2) in B lymphocytes, $\mathrm{T}$ lymphocytes, NK cells, DCs, macrophages, monocytes, neutrophils, eosinophils, basophils, mastocytes, platelets, megakaryocytes, and red blood cells. Our data showed that FCGR2A expression was correlated with the expression of 21 immune markers in the HNSC samples. In the adjacent cancer tissues, the expression of FCGR2A was correlated with 16 immune markers.

3.5. Roles of FCGR2A Gene in the Evaluation of HNSC Prognosis. In this section, PROGgene V2 was used for the correlation between the FCGR2A gene and the distal metastasis or local recurrence in the HNSC samples. These data indicated a correlation between the FCGR2A gene and distal HNSC metastasis $(P=0.012)$. In contrast, there was no correlation between local recurrence and the expression of the FCGR2A gene $(P=0.13$, Figure 11).

Subsequently, the LOGpc database was used for analyzing the survival of HNSC patients, followed by analysis of TCGA data. This indicated that the FCGR2A gene expression was correlated with survival and prognosis (Figure 12). To be specific, there were statistical differences in the survival probability between the high FCGR2A mRNA samples and low FCGR2A mRNA samples. Meanwhile, the GSE65858 dataset was selected for the validation. The FCGR2A expression was significantly correlated with total survival $(P=0.0107)$ and progression-free survival ( $P=0.0362$, Figure 13). This further validated that expression of FCGR2A was correlated with survival and prognosis of HNSC patients.

\section{Discussion}

A majority of HNSC patients are diagnosed at the locally advanced stages of III and IV [15]. In clinical settings, the main treatment option of HNSC is the combination of surgical resection and adjuvant radiotherapy, or concurrent radiotherapy and chemotherapy [16, 17]. Tumor staging and pathology analysis are crucial for the prognostic prediction, which is also an important basis for the guidance of the optimal adjuvant therapy. To date, there are no specific biomarkers for therapeutic strategies of HNSC. Fortunately, several biomarkers have been developed as potential factors to predict and evaluate the prognosis of patients. For instance, the $\mathrm{Wnt} / \beta$-catenin pathway could inhibit cell apoptosis and promote the growth of squamous cell carcinoma. Additionally, the $\mathrm{Wnt} / \beta$-catenin pathway was regulated by a variety of antagonistic genes that were differentially expressed in HNSC. This demonstrated a relationship with therapeutic sensitivity, which may serve as a potential biomarker for treatment and prognosis of HNSC $[16,18]$. To date, there are still some disputes on the prognostic prediction of these biomarkers [19]. Thus, identification of independent markers associated with the prognosis of HNSC is conducive to their clinical application.

The FCGR2A gene encodes a member of a family of immunoglobulin Fc receptor genes that was identified on the surface of many immune response cells [20]. The FCGR2A protein is considered as a cell surface receptor on phagocytic cells (e.g., macrophages and neutrophils), which is involved in the process of phagocytosis [21]. According to the previous description, FCGR2A was mainly reported to bind with the IgG and was expressed in the neutrophils, monocytes, macrophages, and DCs [22]. Fc-gamma receptor polymorphisms were associated with efficacy but not with toxicity in wild-type KRAS, cetuximab-treated colorectal 
TABLE 2: Correlation between FCGR2A and immune markers of various immune cells in HNSCC tumor samples and adjacent tissues.

\begin{tabular}{|c|c|c|c|c|c|}
\hline \multirow{2}{*}{ Immunocytes } & \multirow{2}{*}{ Immune-labeled genes } & \multicolumn{2}{|c|}{ HNSCC tissues } & \multicolumn{2}{|c|}{ Adjacent HNSCC tissues } \\
\hline & & $P$ value & $R$ & $P$ value & $R$ \\
\hline \multirow[t]{2}{*}{ B lymphocytes } & CD19 & 0.45 & -0.033 & 0.038 & 0.31 \\
\hline & $\mathrm{CD} 2$ & $5.70 E-13$ & 0.31 & 0.22 & 0.19 \\
\hline \multirow[t]{3}{*}{ T lymphocytes } & $\mathrm{CD} 3 \mathrm{D}$ & $6.60 E-06$ & 0.2 & 0.14 & 0.22 \\
\hline & CD3E & $6.90 E-11$ & 0.28 & 0.22 & 0.19 \\
\hline & CD56 & $1.70 E-05$ & 0.19 & 0.18 & 0.2 \\
\hline \multirow[t]{2}{*}{ NK cells } & CD94 & $4.20 E-14$ & 0.32 & 0.006 & 0.41 \\
\hline & NKp46 & $3.60 E-07$ & 0.22 & 0.035 & 0.32 \\
\hline \multirow[t]{2}{*}{ Dendritic cells } & CD11c & 0 & 0.52 & $1.30 E-14$ & 0.87 \\
\hline & CD11b & $1.80 E-07$ & 0.23 & $6.20 E-13$ & 0.84 \\
\hline \multirow[t]{2}{*}{ Macrophages } & CD68 & 0 & 0.62 & $1.70 E-07$ & 0.69 \\
\hline & CD163 & 0 & 0.78 & $1.20 E-10$ & 0.79 \\
\hline \multirow[t]{2}{*}{ Monocytes } & CD14 & 0 & 0.73 & $5.10 E-10$ & 0.78 \\
\hline & CD116 & $1.80 E-07$ & 0.23 & $6.20 E-13$ & 0.84 \\
\hline \multirow[t]{3}{*}{ Neutrophils } & $\mathrm{CD} 44$ & $8.90 E-10$ & 0.26 & 0.12 & 0.24 \\
\hline & CD55 & 0.87 & -0.007 & 0.14 & 0.23 \\
\hline & CD45 & 0 & 0.52 & $2.80 E-06$ & 0.64 \\
\hline \multirow[t]{2}{*}{ Eosinophils } & CD125 & 0.0012 & 0.14 & 0.97 & 0.0061 \\
\hline & CD193 & $1.30 E-14$ & 0.33 & 0.45 & 0.12 \\
\hline \multirow{3}{*}{ Basophils } & $\mathrm{CD} 22$ & 0.99 & -0.00075 & 0.00064 & 0.49 \\
\hline & CD45 & 0 & 0.52 & $2.80 E-06$ & 0.64 \\
\hline & CD33 & 0 & 0.49 & $2.40 E-11$ & 0.81 \\
\hline \multirow[t]{3}{*}{ Mastocytes } & CD117 & 0.00021 & 0.26 & $9.10 E-11$ & 0.8 \\
\hline & CD203c & 0.065 & 0.081 & 0.0022 & 0.45 \\
\hline & CD41 & 0.028 & -0.096 & 0.78 & 0.043 \\
\hline \multirow{3}{*}{ Platelets } & CD42a & 0.064 & 0.081 & 0.6 & 0.082 \\
\hline & $\mathrm{CD} 42 \mathrm{~b}$ & 0.7 & 0.017 & 0.0032 & 0.43 \\
\hline & CD61 & 0 & 0.45 & 0.00067 & 0.49 \\
\hline Megakaryocyte & $\mathrm{CD} 41 \mathrm{~b}$ & 0.028 & -0.096 & 0.78 & 0.043 \\
\hline Red blood cells & $\mathrm{CD} 235 \mathrm{a}$ & 0.27 & -0.049 & 0.32 & 0.15 \\
\hline
\end{tabular}

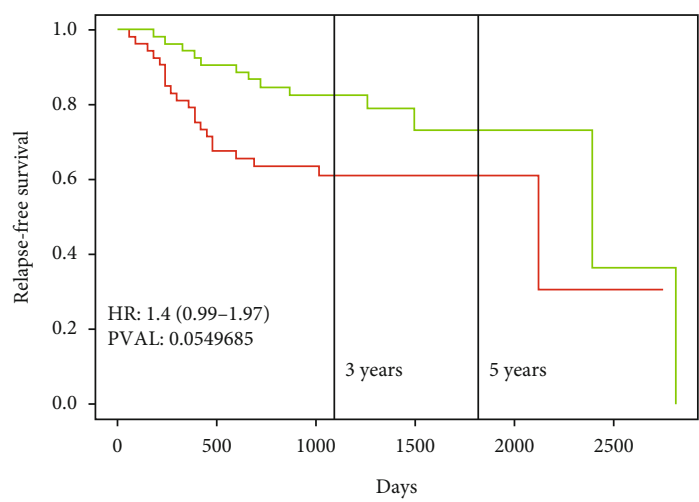

(a)

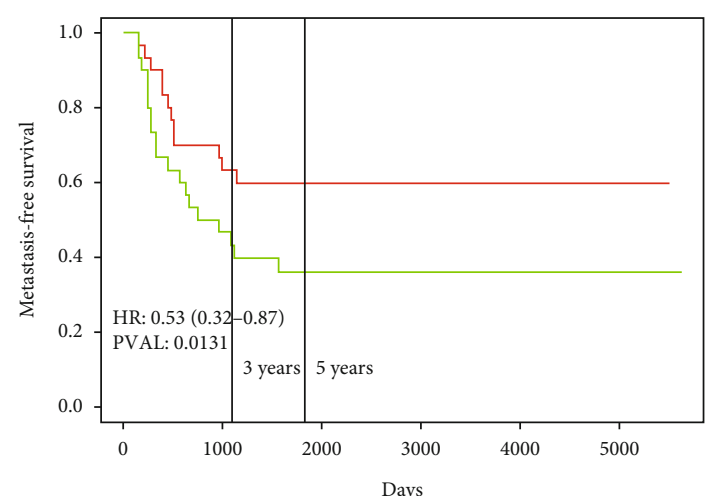

(b)

FIGURE 11: FCGR2A gene expression and recurrence or metastasis curve in HNSC patients. (a) Recurrence curve. (b) Metastasis curve. Red: high gene expression; green: low gene expression. 


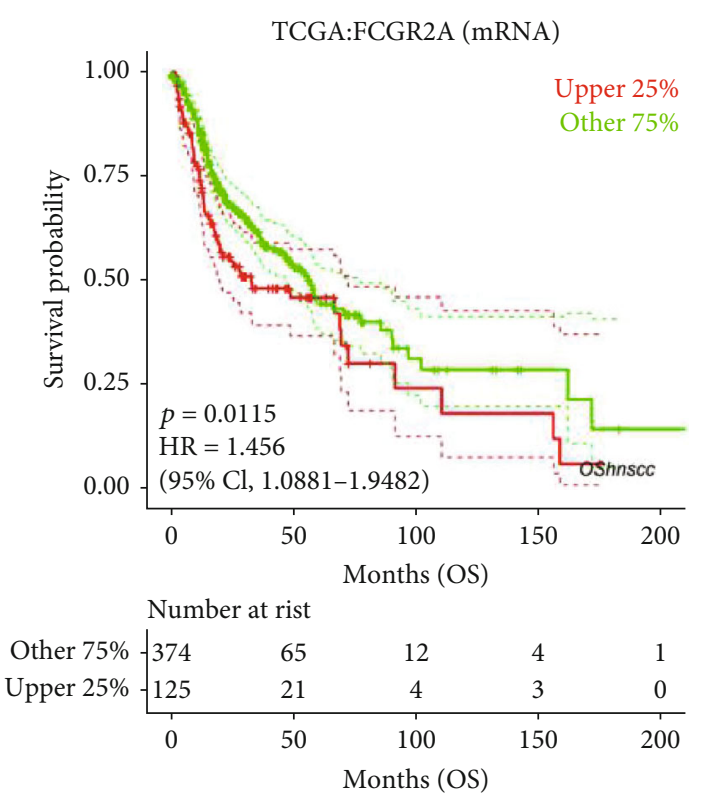

(a)
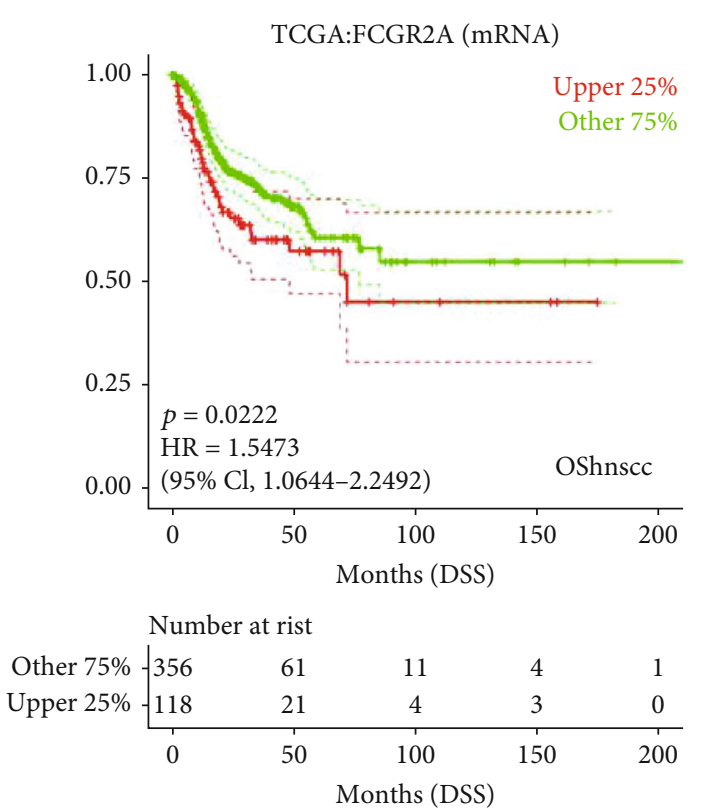

(b)

FIGURE 12: Survival curve of FCGR2A gene and HNSC in TCGA samples. (a) Overall survival (OS) curve. (b) Disease-specific survival (DSS) curve.

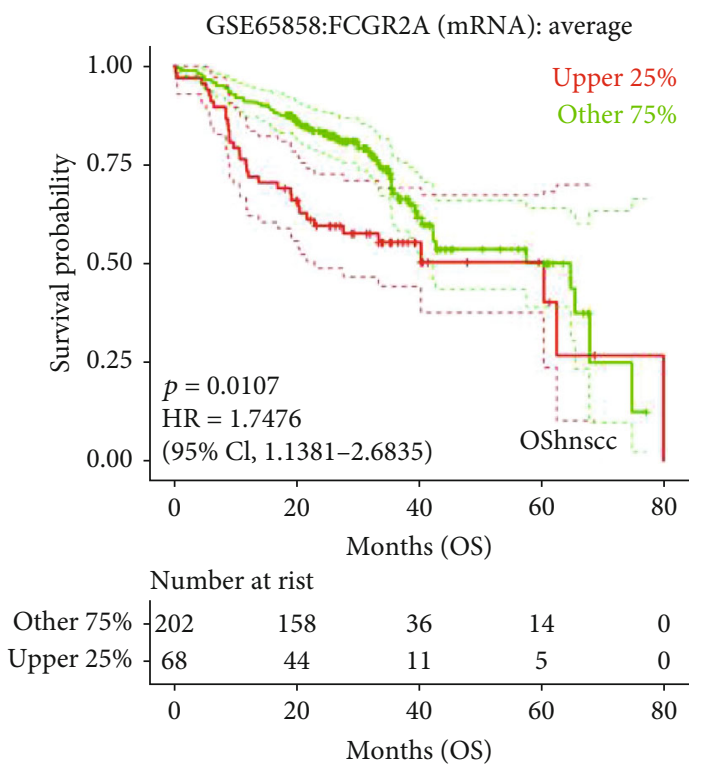

(a)

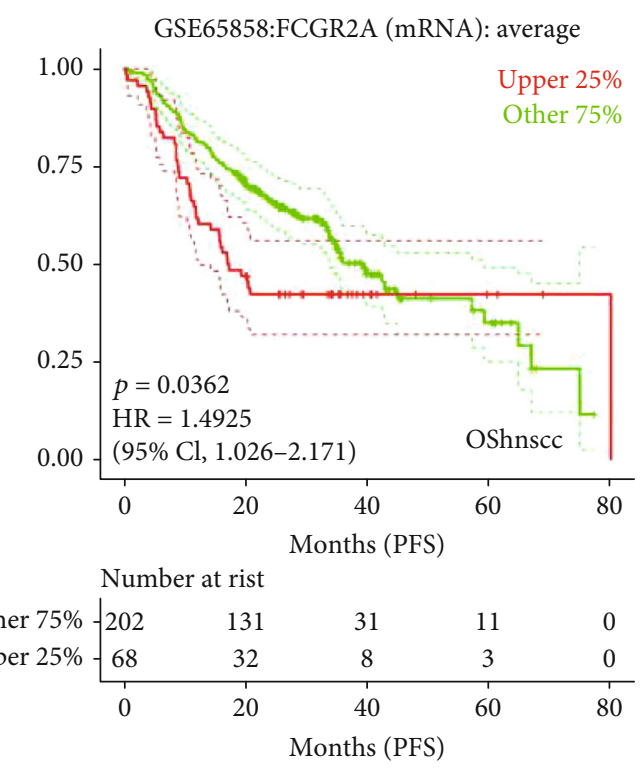

(b)

FIGURE 13: Survival curve of FCGR2A gene and head and neck cancer in GSE65858 data. (a) Overall survival (OS) curve. (b) Progression-free survival (PFS) curve.

cancer patients. Additionally, in a study focused on the exploration of the influence of genetic variants on susceptibility to chronic periaortitis, Alberici et al. reported that FCGR2A was a gene hallmarker for the autoimmunity among the patients with chronic periaortitis [20]. Moreover, in a study that investigated the association of CD1 and FCGR2A gene polymorphisms with Guillain-Barré syndrome susceptibility, Zhang et al. indicated that FCGR2A gene polymorphisms may contribute to GBS risk in Cauca- sians and revealed a certain trend toward significance in the association of the exon 2 of the CD1E gene with GBS in Caucasians [23]. Up to now, there are few studies focusing on the expression of FCGR2A among HNSC patients. Our data showed that FCGR2A expression was correlated with the expression of 21 immune markers in the HNSC samples, while in the adjacent cancer tissues, the expression of FCGR2A was correlated with 16 immune markers. This contributed to the illustration on the roles of the FCGR2A 
gene in radiotherapy resistance among carcinomas of nasopharynx patients.

The circulating FCGR2A gene was comparatively higher than that of the other tissues. In the cellular level, the FCGR2A gene was mainly expressed on the Golgi body and the cell membrane. As revealed by the GEPIA website, FCGR2A level in partial cancer tissues was comparatively higher than the adjacent normal tissues, including carcinoma of bile duct, esophagus carcinoma, clear cell carcinoma of kidney, and gastric cancer. In the HNSC patients with HPV negativity, the expression of FCGR2A in cancer tissues was higher than that of the adjacent tissues. Paradoxically, in certain types of tumor, the expression of FCGR2A in the cancer tissues was significantly lower than the adjacent tissues. To date, little is known about the association between the expression of FCGR2A and the prognosis of these patients, as most of the studies focused on the FCGR2A polymorphism. In our study, the expression of FCGR $2 A$ mRNA and protein was reported to be associated with the tumor staging from grade 1 to grade 3 . Interestingly, in those with grade 4 cancer, the expression of FCGR2A showed a decline in all the 7 cases. This may not present a trend as the sample size is not adequate. Besides, our data indicated that there was no correlation between FCGR2A expression and gender, race, and tumor staging.

In this study, we screened the top 10 proteins associated with FCGR2A, which formed a potential protein interaction network involving the activation of the FCGR signaling pathway. After network analysis, these proteins were involved in FCGR activation, immunological response activation, phagocytosis, PI3KCI signaling pathway, PTP1B signaling pathway, integrin-mediated signaling pathway, CD28 family cosimulation, and bacterial response. According to the GBM/OV microarray database in the Timer website, the expression of FCGR2A was associated with the function of 6 types of immunocytes. Then, the TIMER databases were employed to investigate the correlation between the expression of FCGR2A in the immunocytes of the HNSC patients and the expression of immune marker genes, which indicated that the FCGR2A gene may be involved in the immune infiltration of the HNSC. In addition, infiltration of tumor immunocytes refer to a process in which immunocytes are transmitted to the cancer tissues through circulation. On this basis, it was possible to isolate the infiltrated immunocytes from the cancer tissues. According to a previous study, the infiltration of immunocytes in cancer tissues was considered to be related to the clinical outcomes in cancer patients. Therefore, infiltration of immunocytes in cancer tissues may serve as a drug target in clinical treatment.

PROGgeneV2 is a software displaying the prognostic implications of genes in various cancers. To our best knowledge, most of the studies on FCGR2A have been focused on the inflammation and cerebral vascular diseases. For instance, in a recent study, there was variation in the expression of the FCGR2A gene in the platelets after onset of myocardial infarction [24]. Besides, FCGR2A single nucleotide polymorphism conferred susceptibility to childhood-onset idiopathic nephrotic syndrome [25]. Moreover, there was genetic variation of human neutrophil $\mathrm{F} c \gamma$ receptors and
$\operatorname{SIRP} \alpha$ in antibody-dependent cellular cytotoxicity toward cancer cells [26]. In this study, it was used to investigate the correlation of FCGR2A gene expression and the distal metastasis and local occurrence of HNSC. The results showed that the FCGR2A gene was correlated with distal metastasis rather than local recurrence in HNSC patients. Subsequently, the LOGpc database was utilized to investigate the correlation between FCGR2A gene expression and survival of HNSC patients. These data indicated that FCGR2A gene expression was associated with the survival and prognosis of the HNSC patients. Based on the validation using GSE65858, there was a significant difference in the FCGR2A expression and the total survival rate of HNSC cases. Meanwhile, there was a significant association between FCGR2A expression and progression-free survival. This further validated the correlation between FCGR2A expression and the survival prognosis among HNSC patients. All these implied that FCGR2A may serve as an effective factor for the prediction of prognosis of HNSC.

There are some limitations in our study. We only predicted the correlation between FCGR2A mRNA in tumor immunology and its prognostic value based on online tools. In future, experimental studies are required to further illustrate the exact role of FCGR2A mRNA via downregulating or upregulating its expression.

\section{Conclusions}

In summary, our study provided insights into understanding the potential role of FCGR2A in tumor immunology and its prognostic value. FCGR2A mRNA level was correlated with prognosis and immune infiltrating levels in HNSC, which indicated that it could be used as a prognostic biomarker. In the future, further studies are required to evaluate the potency of the FCGR2A inhibitor interfering with immune cells.

\section{Data Availability}

The datasets used for the current study are available upon reasonable request from the corresponding author.

\section{Ethical Approval}

The authors state that they have obtained appropriate institutional review board approval or have followed the principles outlined in the Declaration of Helsinki in their investigations.

\section{Consent}

Each patient signed the informed consent.

\section{Conflicts of Interest}

The authors declare that they have no competing interests.

\section{Authors' Contributions}

DYM wrote the manuscript. CWH, HJP, and performed data collection. DYM and HJP performed data analysis and wrote 
the manuscript. CTJ conceptualized the study and gave final approval for the submission. Yongmei Dai, Wenhan Chen, and Junpeng Huang contributed equally to this work.

\section{Acknowledgments}

This work was supported by the Funding Project of the Fujian Medical University College Student Innovation and Entrepreneurship Training Program (Grant No. C19071).

\section{References}

[1] C. R. Leemans, P. J. F. Snijders, and R. H. Brakenhoff, "The molecular landscape of head and neck cancer," Nature Reviews. Cancer, vol. 18, no. 5, pp. 269-282, 2018.

[2] F. Bray, J. Ferlay, I. Soerjomataram, R. L. Siegel, L. A. Torre, and A. Jemal, "Global cancer statistics 2018: GLOBOCAN estimates of incidence and mortality worldwide for 36 cancers in 185 countries," CA: A Cancer Journal for Clinicians, vol. 68, no. 6, pp. 394-424, 2018.

[3] F. Lemaire, R. Millon, J. Young et al., "Differential expression profiling of head and neck squamous cell carcinoma (HNSCC)," British Journal of Cancer, vol. 89, no. 10, pp. 1940-1949, 2003.

[4] S. V. Bratman, J. P. Bruce, B. O’Sullivan et al., "Human papillomavirus genotype association with survival in head and neck squamous cell carcinoma," JAMA Oncology, vol. 2, no. 6, pp. 823-826, 2016.

[5] B. K. Flesch, F. Bauer, and J. Neppert, "Rapid typing of the human Fc gamma receptor IIA polymorphism by polymerase chain reaction amplification with allele-specific primers," Transfusion, vol. 38, no. 2, pp. 174-176, 1998.

[6] I. M. van der Meer, J. C. Witteman, A. Hofman, C. Kluft, and M. de Maat, "Genetic variation in Fc $\gamma$ receptor IIa protects against advanced peripheral atherosclerosis," Thrombosis and Haemostasis, vol. 92, no. 12, pp. 1273-1276, 2004.

[7] G. Saruhan-Direskeneli, T. Hughes, K. Aksu et al., "Identification of multiple genetic susceptibility loci in Takayasu arteritis," American Journal of Human Genetics, vol. 93, no. 2, pp. 298-305, 2013.

[8] K. S. Kwon, H. Y. Cho, and Y. J. Chung, "Recapitulation of candidate systemic lupus erythematosus-associated variants in Koreans," Genomics \& Informatics, vol. 14, no. 3, pp. 8589, 2016.

[9] S. L. Xia, D. P. Lin, Q. R. Lin et al., "A case-control study on association of ulcerative colitis with FCGR2A gene polymorphisms in Chinese patients," Genetic Testing and Molecular Biomarkers, vol. 22, no. 10, pp. 607-614, 2018.

[10] D. Shepshelovich, A. R. Townsend, O. Espin-Garcia et al., "Fcgamma receptor polymorphisms, cetuximab therapy, and overall survival in the CCTG CO.20 trial of metastatic colorectal cancer," Cancer Medicine, vol. 7, no. 11, pp. 5478-5487, 2018.

[11] B. Li, E. Severson, J. C. Pignon et al., "Comprehensive analyses of tumor immunity: implications for cancer immunotherapy," Genome Biology, vol. 17, no. 1, p. 174, 2016.

[12] B. Li and J. Z. Li, "A general framework for analyzing tumor subclonality using SNP array and DNA sequencing data," Genome Biology, vol. 15, no. 9, p. 473, 2014.

[13] G. X. Hou, P. Liu, J. Yang, and S. Wen, "Mining expression and prognosis of topoisomerase isoforms in non-small-cell lung cancer by using Oncomine and Kaplan-Meier plotter," PLoS One, vol. 12, no. 3, article e0174515, 2017.

[14] K. Kashofer, E. Winter, I. Halbwedl et al., "HPV-negative penile squamous cell carcinoma: disruptive mutations in the TP53 gene are common," Modern Pathology, vol. 30, no. 7, pp. 1013-1020, 2017.

[15] S. Tanadini-Lang, P. Balermpas, M. Guckenberger et al., "Radiomic biomarkers for head and neck squamous cell carcinoma," Strahlentherapie und Onkologie, vol. 196, no. 10, pp. 868-878, 2020.

[16] B. Solomon, R. J. Young, and D. Rischin, "Head and neck squamous cell carcinoma: genomics and emerging biomarkers for immunomodulatory cancer treatments," Seminars in Cancer Biology, vol. 52, Part 2, pp. 228-240, 2018.

[17] E. Tang, L. Lahmi, N. Meillan, G. Pietta, S. Albert, and P. Maingon, "Treatment strategy for distant synchronous metastatic head and neck squamous cell carcinoma," Current Oncology Reports, vol. 21, no. 11, p. 102, 2019.

[18] R. Galot, C. le Tourneau, J. Guigay et al., "Personalized biomarker-based treatment strategy for patients with squamous cell carcinoma of the head and neck: EORTC position and approach," Annals of Oncology, vol. 29, no. 12, pp. 23132327, 2018.

[19] T. Takeuchi, H. Kawasaki, A. Luce et al., "Insight toward the microRNA profiling of laryngeal cancers: biological role and clinical impact," International Journal of Molecular Sciences, vol. 21, no. 10, p. 3693, 2020.

[20] F. Alberici, F. Bonatti, F. Maritati et al., "Association of a polymorphism of the Fc $\gamma$-receptor 2A (FCGR2A) gene with chronic periaortitis," Clinical and Experimental Rheumatology, vol. 37, no. 2, pp. 222-226, 2018.

[21] L. W. Treffers, M. van Houdt, C. W. Bruggeman et al., "Fc $\gamma$ RIIIb restricts antibody-dependent destruction of cancer cells by human neutrophils," Frontiers in Immunology, vol. 9, p. 3124, 2018.

[22] H. Moriya, K. Saito, N. Helsby et al., "Single-nucleotide polymorphisms and copy number variations of the FCGR2A and FCGR3A genes in healthy Japanese subjects," Biomedical Reports, vol. 2, no. 2, pp. 265-269, 2014.

[23] L. Zhang, L. Liu, H. Li, L. Guo, Q. Yu, and J. Teng, “Association of $\mathrm{CD} 1$ and $\mathrm{Fc} \gamma \mathrm{R}$ gene polymorphisms with Guillain-Barré syndrome susceptibility: a meta-analysis," Neurological Sciences, vol. 39, no. 12, pp. 2141-2149, 2018.

[24] S. R. McMahon, S. Chava, H. S. Taatjes-Sommer, S. Meagher, K. E. Brummel-Ziedins, and D. J. Schneider, "Variation in platelet expression of Fc $\gamma$ RIIa after myocardial infarction," Journal of Thrombosis and Thrombolysis, vol. 48, no. 1, pp. 88-94, 2019.

[25] G. M. Rossi, F. Bonatti, A. Adorni et al., "FCGR2A single nucleotide polymorphism confers susceptibility to childhood-onset idiopathic nephrotic syndrome," Immunology Letters, vol. 193, pp. 11-13, 2018.

[26] L. W. Treffers, X. W. Zhao, J. van der Heijden et al., "Genetic variation of human neutrophil $\mathrm{Fc} \gamma$ receptors and $\operatorname{SIRP} \alpha$ in antibody-dependent cellular cytotoxicity towards cancer cells," European Journal of Immunology, vol. 48, no. 2, pp. 344-354, 2018. 